PROCEEDINGS OF THE

AMERICAN MATHEMATICAL SOCIETY

Volume 131, Number 4, Pages 1289-1296

S 0002-9939(02)06621-2

Article electronically published on July 25, 2002

\title{
REFLECTING POINT-COUNTABLE FAMILIES
}

\author{
ZOLTAN T. BALOGH
}

(Communicated by Alan Dow)

\begin{abstract}
It is shown that if every $\leq \omega_{1}$-sized subspace of a (regular) space $X$ of density $\leq \omega_{1}$ has a point-countable base, then so does $X$. Similar results hold for meta-Lindelöfness. Dow's reflection theorem and a number of other results are deduced as corollaries and applications.
\end{abstract}

\section{INTRODUCTION}

A well-known reflection theorem of Alan Dow ([D88, [D91) says that if every subspace of cardinality $\leq \omega_{1}$ of a countably compact space $X$ is metrizable, then $X$ is separable metrizable. The aim of this paper is to extend this result to the non-separable case. It is easy to see (cf. the proof of Theorem 3.1) that Dow's theorem is equivalent to showing that if every subspace of cardinality $\leq \omega_{1}$ of a countably compact space of density $\leq \omega_{1}$ has a point-countable base, then so does $X$. It is shown that in this form of Dow's theorem, countable compactness can be dropped (Theorem 1.5). Theorem 1.5 also strengthens some results of Vaughan and Hodel [HV], $\mathrm{V}$.

The paper is divided into four sections. In Section 1, we prove Theorem 1.5 mentioned above. In section 2 we prove analogous results for meta-Lindelöfness (Theorem 2.4 and Corollary 2.7). The proofs also include an analysis that shows that it is enough to make the hypotheses for special kinds of subspaces of cardinality $\leq \omega_{1}$ we call thin-short (resp. thin or short). In Section 3, a number of corollaries and applications are given. It is also pointed out that the results and the proofs remain valid, word for word, if we replace $\omega_{1}$ with any regular cardinal $\kappa$. Finally, in Section 4, examples showing the sharpness of the results are given and open questions are raised.

Our terminology and notation follow the standards of set-theory and set-theoretic topology as used in $[\mathrm{K}]$ and $[\mathrm{KV}]$. In particular, $[H]^{\kappa},[H]^{\leq \kappa}$ denote the family of subsets of $H$ of cardinality $\kappa(\leq \kappa)$.

By a space we mean regular $T_{1}$-space in this paper. "An elementary submodel of $H(\lambda)$ " includes the assumption that $\lambda$ is big enough for the whole proof to take place in $H(\lambda)$.

Received by the editors July 31, 2001 and, in revised form, November 2, 2001.

2000 Mathematics Subject Classification. Primary 54E35, 54A35, 54D20.

Key words and phrases. Point-countable, reflection.

(C)2002 American Mathematical Society 


\section{Reflecting POINT-COUNTABle BASES}

Definition 1.1. A space $Y$ is called short if $|Y| \leq \omega_{1}$ and $Y$ is separable. $Y$ is thin if $|Y| \leq \omega_{1}$ and every separable subspace of $Y$ is countable. $Y$ is thin-short if $Y$ is the union of a thin and a short subspace. $Y$ is thin or short if $Y$ is either thin or short.

Lemma 1.2. Let $X$ be a space, and let $D \in[X] \leq \omega_{1}$ be such that $X=\bigcup\{\bar{A}$ : $\left.A \in[D]^{\omega}\right\}$. Let $M_{0}=\emptyset$, and let $\left\langle M_{\alpha}\right\rangle_{0<\alpha<\omega_{1}}$ be a continuous $\epsilon$-chain of countable elementary submodels of $H(\lambda)$ such that $X, D$ and the topology $\mathcal{T}$ of $X$ are all elements of $M_{1}$. Suppose that there is a c.u.b. $C \subset \omega_{1}$ such that for every $\alpha \in$ $C, \mathcal{T} \cap M_{\alpha}$ contains a base at every point of $\overline{X \cap M_{\alpha}}$. Then $X$ has a point-countable base.

Proof. By passing to an increasing enumeration of $C$, we can assume without loss of generality that $C=\omega_{1}$. Then for every $\beta \in \omega_{1}$, let us set

$$
\mathcal{B}_{\beta}=\left\{U \backslash \overline{X \cap M_{\beta}}: U \in \mathcal{T} \cap M_{\beta+1}\right\} .
$$

We will show that $\mathcal{B}=\bigcup_{\beta \in \omega_{1}} \mathcal{B}_{\beta}$ is a point-countable base for $X$. To prove that $\mathcal{B}$ is point-countable, let $x \in X$. Set $M=\bigcup_{\alpha \in \omega_{1}} M_{\alpha}$. Since $D \in M_{1} \subset M$ and $|D| \leq \omega_{1}$, it follows that $D \subset X \cap M$. Thus $X=\bigcup_{\beta \in \omega_{1}}{\overline{X \cap M_{\beta}}}_{\beta}$, so there is a $\beta \in \omega_{1}$ such that $x \in \overline{X \cap M_{\beta}}$. Then $x \in B \in \mathcal{B}$ implies $B \in \bigcup_{\alpha<\beta} \mathcal{B}_{\alpha}$.

To prove that $\mathcal{B}$ forms a base of $X$, let $x \in V \in T$, and pick the smallest $\alpha \in \omega_{1}$ with $x \in \overline{X \cap M_{\alpha}}$. Note that by $M_{0}=\emptyset, \alpha \geq 1$. We consider two cases.

Case 1. If $\alpha=\beta+1$ is a successor ordinal, then by $x \in \overline{X \cap M_{\alpha}}$ there is a $U \in \mathcal{T} \cap M_{\beta+1}$ such that $x \in U \subset V$. Since $\alpha$ is minimal for $x \in \overline{X \cap M_{\alpha}}$, it follows that $x \notin \overline{X \cap M_{\beta}}$. Thus for $B=U \backslash \overline{X \cap M_{\beta}} \in \mathcal{B}_{\beta} \subset \mathcal{B}$ we have $x \in B \subset V$.

Case 2. If $\alpha$ is a limit ordinal, then there is a $U \in \mathcal{T} \cap M_{\alpha}$ such that $x \in U \subset V$. Since $\alpha$ is a limit, there is $\beta<\alpha$ such that $U \in \mathcal{T} \cap M_{\beta+1}$.

By the minimality of $\alpha$, it follows that $x \notin \overline{X \cap M_{\beta}}$. Thus for $B=U \backslash \overline{X \cap M_{\beta}} \in$ $\mathcal{B}_{\beta} \subset \mathcal{B}$ we have $x \in B \subset V$.

Proposition 1.3. Suppose $X$ is a space of density $\leq \omega_{1}$ with topology $\mathcal{T}$ such that every thin subspace of $X$ is first countable, and let $M$ be an elementary submodel of $H(\lambda)$ with $X, \mathcal{T} \in M$. Then

(a) $X$ is first-countable;

(b) $\mathcal{T} \cap M$ contains a base at every point of $X \cap M$.

Proof. (a) Let $x \in X$. Pick a dense subspace $D$ of $X$ which is left-separated in type $\leq \omega_{1}$. Then $Y=D \cup\{x\}$ is thin, so it is first countable. Since $X$ is regular, $\chi(x, X)=\chi(x, Y)=\omega$.

(b) Let $x \in X \cap M$. Since there is a countable base $\mathcal{V} \subset \mathcal{T}$ at $x$, there is such a $\mathcal{V} \in M$. Since $\mathcal{V}$ is countable, $\mathcal{V} \subset \mathcal{T} \cap M$.

Lemma 1.4. Suppose $X$ is a space such that $d(X) \leq \omega_{1}$ and every thin-short subspace of $X$ has a point-countable base. Then for every separable subspace $Z$ of $X$, there is a countable family $Z$ of open subsets of $X$ such that $Z$ contains a base at every point of $Z$.

Proof. Let $\left\langle M_{\alpha}\right\rangle_{\alpha<\kappa}$ be a continuous $\epsilon$-chain of countable elementary submodels of $H(\lambda)$ such that $X$, the topology $\mathcal{T}$ of $X, Z$ and a countable dense subset $A$ of $Z$ are all elements of $M_{0}$. Assume indirectly that there is no $Z$ as in the Lemma. Then 
for every $\alpha \in \omega_{1}$, there is a $z_{\alpha} \in Z \cap M_{\alpha+1}$ such that $\mathcal{T} \cap M_{\alpha}$ does not form a local base at $z_{\alpha}$. Note that $Z_{1}=A \cup\left\{z_{\alpha}: \alpha \in \omega_{1}\right\}$ is a short subspace of $X$. Let $D$ be a dense subset of $X \backslash \bar{Z}$ which is left-separated in type $\leq \omega_{1}$. Then $Y=Z_{1} \cup D$ is a thin-short subspace, and therefore $Y$ has a point-countable base $\mathcal{B}$. Since $Z_{1}$ is separable $\mathcal{B}_{1}=\left\{B \in \mathcal{B}: B \cap Z_{1} \neq \emptyset\right\}$ is countable and it contains a base in $Y$ at every point of $Z$. Since $Y$ is dense $\mathcal{B}_{2}=\left\{\operatorname{int}(\bar{B}): B \in \mathcal{B}_{1}\right\}$ contains a base in $X$ at every point of $Z$. Let $M=\bigcup_{\alpha \in \omega_{1}} M_{\alpha}$. By Proposition 1.3, $\mathcal{T} \cap M$ contains a base at every point of $Z_{1} \subset X \cap M$. Thus there is a countable $\mathcal{B}_{3} \subset \mathcal{T} \cap M$ such that $\mathcal{B}_{3}$ contains a base at every point of $Z_{1}$. Pick $\alpha \in \omega_{1}$ with $\mathcal{B}_{3} \subset \mathcal{T} \cap M_{\alpha}$. Then $\mathcal{T} \cap M_{\alpha}$ contains a base at every point of $Z_{1}$, contrary to the definition of $z_{\alpha} \in Z_{1}$.

Theorem 1.5. Let $X$ be a space with $d(X) \leq \omega_{1}$ and suppose that every thin-short subspace of $X$ has a point-countable base. Then $X$ has a point-countable base.

Proof. Let $\left\langle M_{\alpha}\right\rangle_{\alpha \in \omega_{1}}$ be as in Lemma 1.2 and set $M=\bigcup_{\alpha \in \omega_{1}} M_{\alpha}$. Recall that $X \cap M$ is dense in $X$. By Proposition 1.3(a), (b), $X$ is first countable (and thus, $t(x)=\omega)$ and $\mathcal{T} \cap M$ contains a base at every point of $X \cap M$. Assume indirectly that $X$ does not have a point-countable base. Then by Lemma 1.2, we can pick a stationary $S \subset \omega_{1}$ such that for every $\alpha \in S$, there is a point $x_{\alpha} \in \overline{X \cap M_{\alpha}}$ such that $\mathcal{T} \cap M_{\alpha}$ does not contain a base at $x_{\alpha}$. Since $\overline{X \cap M_{\alpha}}, \mathcal{T} \cap M_{\alpha} \in M_{\alpha+1}$ we can pick such $x_{\alpha} \in M_{\alpha+1}$. We will prove that

$$
\beta<\alpha \text { implies } x_{\alpha} \notin \overline{X \cap M_{\beta}} \text {. }
$$

Indeed, by Lemma 1.4 , there is a countable $Z \subset \mathcal{T}$ such that $Z$ contains a base at every point of $Z=\overline{X \cap M_{\beta}}$. Since $\mathcal{T}, Z \in M_{\alpha}$, there is such a $Z \in M_{\alpha}$. Since $Z$ is countable, $Z \subset \mathcal{T} \cap M_{\alpha}$, so $\mathcal{T} \cap M_{\alpha}$ contains a base at every point of $\overline{X \cap M_{\beta}}$. Thus $x_{\alpha} \notin \overline{X \cap M_{\beta}}$.

Since $X$ is first countable, it follows that $X=\overline{X \cap M}=\bigcup_{\alpha<\omega_{1}} \overline{X \cap M_{\alpha}}$. Let us take a subset $L$ of $X \cap M$ such that for every $\alpha \in \omega_{1}, L \cap M_{\alpha}$ is dense in $X \cap M_{\alpha}$ and $\left(L \backslash M_{\alpha}\right) \cap \overline{X \cap M_{\alpha}}=\emptyset$. Let us set $Y=L \cup\left\{x_{\alpha}: \alpha \in S\right\}$.

Next, let $\left\langle N_{\gamma}\right\rangle_{\gamma \in \omega_{1}}$ be another continuous $\epsilon$-chain of elementary submodels of $H(\lambda)$ such that $L,\left\langle x_{\alpha}\right\rangle_{\alpha \in \omega_{1}},\left\langle M_{\alpha}\right\rangle_{\alpha \in \omega_{1}} \in N_{0}$. Since $\left\{N_{\gamma} \cap \omega_{1}: \gamma \in \omega_{1}\right\}$ is a c.u.b. subset of $\omega_{1}$, there is a $\gamma \in \omega_{1}$ such that $\delta=N_{\gamma} \cap \omega_{1} \in S$. Let us introduce the notation $N=N_{\gamma}$. Note that for every $A \in M \cap N, A \cap M \cap N=A \cap M_{\delta}$. Since $X, \mathcal{T} \in N_{0} \cap M \subset N \cap M$, it follows that $X \cap M \cap N=X \cap M_{\delta}$ and $\mathcal{T} \cap M \cap N=\mathcal{T} \cap M_{\delta}$. Thus $x_{\delta} \in \overline{X \cap M \cap N}$ and $\mathcal{T} \cap M \cap N$ does not contain a base at $x_{\delta}$ in contradiction with the following.

Claim. $\mathcal{T} \cap M \cap N$ contains a base at every point of $E=\overline{X \cap M \cap N} \cap Y$.

To prove the Claim note first that by $(*), Y$ is a thin subspace of $X$. Thus $Y$ has a point-countable base.

Since $Y$ is dense in $X$, this implies that there is a $\mathcal{B} \subset \mathcal{T}$ such that $\mathcal{B}$ is pointcountable and contains a base (in $X$ ) at every point of $Y$. Since $X, \tau, Y \in N_{0} \subset N$ we can pick and fix such a $\mathcal{B} \in N$.

Now let $z \in E$ and let $V \ni Z$ be an open set. Since $z \in Y$ there is a $B_{1} \in \mathcal{B}$ such that $Z \in \beta_{1} \subset V$. By Proposition 1.3(b), there is a $U \in M \cap \mathcal{T}$ such that $z \in U \subset B_{1}$. Finally, there is a $B_{2} \in B$ with $z \in B_{2} \subset U$. Since $z \in \overline{X \cap M \cap N}$ and since by $Y \in N, Y \cap N$ is dense in $X \cap M \cap N$, it follows that there is a $y \in B_{1} \cap B_{2} \cap N \cap Y$. Since $B, y \in N, B_{y}=\{B \in B: y \in B\} \in N$. By countability of $B_{y}$ it follows that $B_{y} \subset N$ and thus $B_{1}, B_{2} \in N$. Since the statement "there is a $U \in M \cap \mathcal{T}\left(B_{1} \subset U \subset B_{2}\right)$ " holds in $H(\lambda)$ and $B_{1}, B_{2}, M \cap \mathcal{T} \in N$, it follows that 
there is such a $U \in M \cap \mathcal{T} \cap N$. Since $z \in U \subset V$ and $V$ was an arbitrary open set containing $Z$, we have proved the claim.

\section{Reflecting Point-COUntable COVERS}

Lemma 2.1. Let $X$ be a space, let $D \in[X] \leq \omega_{1}$ with $X=\bigcup\left\{\bar{A}: A \in[D]^{\omega}\right\}$ and let $\mathcal{U}$ be an open cover of $X$. Let $M_{0}=\emptyset$ and let $\left\langle M_{\alpha}\right\rangle_{1 \leq \alpha<\omega_{1}}$ be a continuous $\epsilon$-chain of elementary submodels of $H(\lambda)$ such that $X, \mathcal{U}, D \in M_{1}$. Suppose that for c.u.b. many $\alpha \in \omega_{1}, \mathcal{U} \cap M_{\alpha}$ covers $\overline{X \cap M_{\alpha}}$. Then $\mathcal{U}$ has a point-countable open refinement.

The proof of Lemma 2.1 is very similar to that of Lemma 1.2 and is therefore omitted.

Lemma 2.2. Let $X$ be a space, let $\mathcal{U}$ be an open cover of $X$, and suppose that for every short $Y \subset X$ and every $\mathfrak{U}^{\prime} \subset \mathcal{U}$ with $\bigcup \mathcal{U}^{\prime} \supset Y$ there is an open partial refinement $\mathcal{B}$ of $\mathcal{U}^{\prime}$ such that $\bigcup \mathcal{B} \supset Y$ and $\mathcal{B}$ is point-countable at every point of $Y$. Then every separable subspace $Z \subset X$ is covered by a countable $Z \subset \mathcal{U}$.

Proof. Let $\left\langle M_{\alpha}\right\rangle_{\alpha \in \omega_{1}}$ be a continuous $\epsilon$-chain of elementary submodels of $H(\lambda)$ such that $X, \mathcal{U}$ and a countable dense subset $A$ of $Z$ are all elements of $M_{0}$. Assume indirectly that no countable $Z \subset \mathcal{U}$ covers $Z$. Then for every $\alpha \in \omega_{1}$, there is a point $z_{\alpha} \in Z \backslash \bigcup\left(\mathcal{U} \cap M_{\alpha}\right)$. Since $Z, \mathcal{U} \cap M_{\alpha} \in M_{\alpha+1}$ we can assume without loss of generality that $z_{\alpha} \in M_{\alpha+1}$. Let $Y=A \cup\left\{z_{\alpha}: \alpha \in \omega_{1}\right\}$. Note that $Y$ is short and that by $Y \subset X \cap M, Y$ is covered by $\mathcal{U}^{\prime}=\mathcal{U} \cap M$. Thus there is a partial refinement $\mathcal{B}$ of $\mathcal{U} \cap M$ such that $\cup \mathcal{B} \supset Y$ and $\mathcal{B}$ is point-countable at every point of $Y$. Since $Y$ is separable, $\mathcal{B}$ is actually countable. Since $\mathcal{B}$ is a partial refinement of $\mathcal{U} \cap M$, there is a countable $\mathfrak{U}_{1} \subset \mathcal{U} \cap M=\bigcup_{\alpha \in \omega_{1}} \mathcal{U} \cap M_{\alpha}$ such that $\bigcup \mathcal{U}_{1} \supset Y$. Let $\alpha \in \omega_{1}$ be such that $\mathcal{U}_{1} \subset \mathcal{U} \cap M_{\alpha}$. Then $\bigcup\left(\mathcal{U} \cap M_{\alpha}\right) \supset Y$ contrary to $z_{\alpha} \notin Z \backslash \bigcup\left(\mathcal{U} \cap M_{\alpha}\right)$.

Theorem 2.3. Let $X$ be a space, let $D \in[X] \leq \omega_{1}$ be such that $X=\bigcup\{\bar{A}: A \in$ $\left.[D]^{\omega}\right\}$, and let $\mathcal{U}$ be an open cover of $X$. Suppose that for every thin or short subspace $Y$ and for every $\mathfrak{U}^{\prime} \subset \mathcal{U}$ with $\bigcup \mathcal{U}^{\prime} \supset Y$, there is an open partial refinement $\mathcal{B}$ of $\mathcal{U}^{\prime}$ such that $\bigcup \mathcal{B} \supset Y$ and $\mathcal{B}$ is point-countable at every point of $Y$. Then $\mathcal{U}$ has a point-countable open refinement.

Proof. Let $M_{0}=\emptyset$, and let $\left\langle M_{\alpha}\right\rangle_{0<\alpha<\omega_{1}}$ be a continuous $\epsilon$-chain of elementary submodels of $H(\lambda)$ such that $X, \mathcal{U}, D$ and the topology $\tau$ of $X$ are elements of $M_{1}$. Set $M=\bigcup_{\alpha \in \omega_{1}} M_{\alpha}$. Since $D \in M_{1} \subset M$, it follows that $D \subset M$ and thus, $X=\bigcup_{\alpha \in \omega_{1}} \overline{X \cap M_{\alpha}}$. Assume indirectly that $U$ has no point-countable open refinement. Then by Lemma 2.1 , there is a stationary $S \subset \omega_{1}$ such that for every $\alpha \in S$ we can pick a point $x_{\alpha} \in \overline{X \cap M_{\alpha}} \cap M_{\alpha+1}$ which is not covered by $\mathcal{U} \cap M_{\alpha}$. We will prove that

$$
\beta<\alpha \in S \text { implies } x_{\alpha} \notin \overline{X \cap M_{\beta}} \text {. }
$$

Indeed, by Proposition 2.2 , there is a countable subfamily $\mathcal{Z}$ of $\mathcal{U}$ such that $\overline{X \cap M_{\beta}} \subset \bigcup \mathcal{Z}$. Since $\overline{X \cap M_{\beta}}, \mathcal{U} \in M_{\alpha}$, there is such a $z \in M_{\alpha}$. Since $z$ is countable, it follows that $\mathcal{Z} \subset \mathcal{T} \cap M_{\alpha}$. Thus $\bigcup\left(\mathcal{U} \cap M_{\alpha}\right) \supset \overline{X \cap M_{\beta}}$ and (*) follows as $x_{\alpha} \notin \bigcup\left(\mathcal{U} \cap M_{\alpha}\right)$.

Let us now take a subset $L$ of $X \cap M$ such that for every $\alpha \in \omega_{1}, L \cap M_{\alpha}$ is dense in $X \cap M_{\alpha}$ and $\left(L \backslash M_{\alpha}\right) \cap \overline{X \cap M_{\alpha}}=\emptyset$. Set $Y=L \cup\left\{x_{\alpha}: \alpha \in S\right\}$. 
Next, let $\left\langle N_{\gamma}\right\rangle_{\gamma \in \omega_{1}}$ be another continuous $\epsilon$-chain of countable elementary submodels of $H(\lambda)$ such that $L,\left\langle x_{\alpha}\right\rangle_{\alpha \in \omega_{1}},\left\langle M_{\alpha}\right\rangle_{\alpha \in \omega_{1}} \in N_{0}$. Since $\left\{N_{\gamma} \cap \omega_{1}: \gamma \in \omega_{1}\right\}$ is a c.u.b. subset of $\omega_{1}$, there is a $\gamma \in \omega_{1}$ such that $\delta=N_{\gamma} \cap \omega_{1} \in S$. Let us set $N=N_{\gamma}$. Note that for every $A \in M \cap N, A \cap M \cap N=A \cap M_{\delta}$. Since $X, \mathcal{U} \in N_{0} \cap M \subset N \cap M$, it follows that $X \cap M \cap N=X \cap M_{\delta}$ and $\mathcal{U} \cap M \cap N=\mathcal{U} \cap M_{\delta}$. Thus $x_{\delta} \in \overline{X \cap M \cap N}$ and $\mathcal{U} \cap M \cap N$ does not cover $x_{\delta}$ in contradiction with the following.

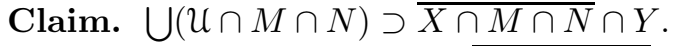

To prove the Claim, let $E=\overline{X \cap M \cap N} \cap Y$. Note first that by $(*), Y$ is a thin subspace of $X$. Thus there is a partial open refinement $\mathcal{B}$ of $\mathcal{U} \cap M$ such that $\cup \mathcal{B} \supset Y$ and $\mathcal{B}$ is point-countable at every point of $Y$.

Let $z \in E$. Since $\bigcup \mathcal{B} \supset Y \supset E$, we can pick a $B \in \mathcal{B}$ with $z \in B$. Note that since $L$ is dense in $X \cap M$ and $L, X \cap M \in N$, it follows that $L \cap N$ is dense in $X \cap M \cap N$. Since $z \in \overline{X \cap M \cap N}$, it follows that there is a point $y \in B \cap L \cap N$. Since $y, \mathcal{B} \in N, \mathcal{B}_{y}=\{B \in \mathcal{B}: y \in B\} \in N$ and since $\mathcal{B}_{y}$ is countable, $B \in \mathcal{B}_{y} \subset N$, i.e. $B \in N$. Since $\mathcal{B}$ is a partial refinement of $\mathcal{U} \cap M$, there is a $U \in \mathcal{U} \cap M$ such that $B \subset \mathcal{U}$. Since $B, \mathcal{U} \in N$, there is such a $\mathcal{U} \in \mathcal{U} \cap M \cap N$. We thus conclude that $z \in \bigcup(U \cap M \cap N)$.

Theorem 2.4. Let $X$ be a space with a $D \in[X] \leq \omega_{1}$ such that $X=\bigcup\{\bar{A}: A \in$ $\left.[D]^{\omega}\right\}$. Suppose that every thin or short subspace of $X$ is meta-Lindelöf. Then $X$ is hereditarily meta-Lindelöf.

Proof. Theorem 2.4 follows directly from Theorem 2.3.

Theorem 2.5. Let $X$ be a space with a $D \in[X] \leq \omega_{1}$ such that $X=\bigcup\{\bar{A}: A \in$ $\left.[D]^{\omega}\right\}$, and let $\mathcal{U}$ be an open cover of $X$ of cardinality $\omega_{1}$. Suppose that for every thin or small $Y \subset X$ there is a partial open refinement $\mathcal{B}$ of $\mathcal{U}$ such that $\bigcup \mathcal{B} \supset Y$ and $\mathcal{B}$ is point-countable at every point of $Y$. Then $\mathcal{U}$ has a point-countable open refinement.

Proof. The proof of Theorem 2.5 is obtained from the proof of Theorem 2.3 by noting that if $|\mathcal{U}|=\omega_{1}$, then $\mathcal{U} \cap M=\mathcal{U}$.

Definition 2.6. $Y \subset X$ is meta-Lindelöf in $X$, if for every open cover $\mathcal{U}$ of $X$, there is a point-countable relatively open cover $\mathcal{H}$ of $Y$ that is a partial refinement of $\mathcal{U}$.

Corollary 2.7. Suppose that $X$ is a space with a $D \in[X] \leq \omega_{1}$ such that $X=$ $\bigcup\left\{\bar{A}: A \in[D]^{\omega}\right\}$ and $L(X)=\omega_{1}$. Suppose that every thin or small $Y \subset X$ is meta-Lindelöf in $X$.

Then $X$ is meta-Lindelöf.

\section{Corollaries and application}

Theorem 3.1. Let $P$ be a closed hereditary property such that for space with property $P$ having a point-countable base implies having a countable base. Let $X$ be a space with property $P$ such that every thin-short subspace of $X$ has a point-countable base. Then $X$ is a separable metrizable space.

Proof. If $d(X) \leq \omega_{1}$, then by Theorem 1.5, $X$ has a point-countable base and is, therefore, second countable.

If $d(X) \geq \omega_{2}$, then let $Z$ be a closed subspace with $d(Z)=\omega_{1}$. Then by Theorem $1.5, Z$ is second countable, contradicting $d(Z)=\omega_{1}$. 
Corollary 3.2. Suppose $X$ is any one of the following kinds of spaces: (a) countably compact; (b) Lindelof p-space. Suppose further that every thin-short subspace of $X$ has a point-countable base. Then $X$ is a separable metrizable space.

Corollary 3.2.1 (Dow, D88, D91]). If $X$ is a countably compact space and every subspace of cardinality $\leq \omega_{1}$ of $X$ has a point-countable base, then $X$ is separable metrizable.

Corollary 3.2.2 (Vaughan, $[\nabla])$. If $X$ has a conditionally compact dense subspace and every subspace of cardinality $\leq \omega_{1}$ of $X$ has a point-countable base, then $X$ is separable metrizable.

Corollary 3.3 (Hodel and Vaughan, [HV]). If $d(X) \leq \omega_{1}$ and every subspace of cardinality $\leq \omega_{1}$ of $X$ has a point-countable base, then $w(X) \leq \omega_{1}$.

Theorem 3.4. Suppose $d(X) \leq \omega_{1}$ and $X$ is one of the following kinds of spaces: (a) locally compact; (b) a paracompact p-space. Suppose that every thin-short subspace of $X$ has a point-countable base. Then $X$ is metrizable.

Proof. By Theorem 1.5, $X$ has a point-countable base which in the given classes of spaces implies metrizability.

Remark. It is a fascinating question whether $d(X)=\omega_{1}$ can be dropped from Theorem 3.4. For the locally compact case, this is independent of ZFC (see Example 4.4 and Theorem 4.3).

One type of potential application of the reflection theorems in this paper is to use forcing or Martin's Axiom to prove that all "small" subspaces are "good" and then use reflection to conclude that the whole space is. From this point of view it is worthwhile to see how Theorem 2.4 plugs in to reprove a result from $B$ (Theorem 3.6 ), even if it only saves a pressing down argument. We need the following result first.

Lemma 3.5 ([B83]). Assume $M A\left(\omega_{1}\right)$. Suppose that $Y$ is a locally countable subspace of size $\omega_{1}$ in a compact space of countable tightness. Then $Y$ is the union of countably many discrete subspaces.

Theorem 3.6 ([B83]). Assume $M A\left(\omega_{1}\right)$. Let $X$ be a locally hereditarily separable, hereditarily collectionwise Hausdorff subspace of a countably tight compact space. Then $X$ is paracompact.

Proof. We will show first that every separable subspace $Z$ of $X$ is hereditarily Lindelöf. Otherwise $Z$ contains a locally countable subspace $Y$ of size $\omega_{1}$. By Theorem 3.5, $Y$ has an uncountable discrete subspace which by hereditarily collectionwise Hausdorff contradicts separability of $Z$.

We conclude that all short subspaces are (hereditarily) Lindelöf. Next, let $Y$ be a thin subspace of $X$. Since $X$ is locally hereditarily separable, it follows that $Y$ is locally countable. Thus $Y$ is the union of countably many discrete subspaces, and by hereditarily collectionwise Hausdorff, $Y$ is meta-Lindelöf. Finally a locally hereditarily separable, hereditarily collectionwise Hausdorff space has density $\leq \omega_{1}$ ([B83]), so Theorem 2.5 applies to conclude that $X$ is meta-Lindelöf. In locally separable spaces, this implies paracompact.

By substituting $<\kappa$ in place of $\omega$ and $\kappa$ in place of $\omega_{1}$ we can repeat, word for word, the proofs in the first two sections to obtain the following results. Let us say that a family of sets is point- $<\kappa$, if no point is in $\geq \kappa$ members. 
Theorem 3.7. Let $\kappa \geq \omega_{1}$ be a regular cardinal. Let $X$ be a space with $d(X) \leq \kappa$ and suppose that every subspace of $X$ of cardinality $\leq \kappa$ has a point $-<\kappa$ base. Then $X$ has a point $-<\kappa$ base.

Let us say that $X$ is meta- $<\kappa$-Lindelöf if every open cover has a point- $<\kappa$ open refinement.

Theorem 3.8. Let $\kappa \geq \omega_{1}$ be a regular cardinal. Let $X$ be a space with a subset $D$ of cardinality $\leq \kappa$ such that $X=\bigcup\left\{\bar{A}: A \in[D]^{<\kappa}\right\}$. Suppose that every subspace of $X$ of cardinality $\leq \kappa$ is meta- $<\kappa$-Lindelöf. Then $X$ is hereditarily meta- $<\kappa$ Lindelöf.

\section{EXAMPLES AND QUESTIONS}

Example 4.1. Let $X=\omega_{2}+1$, and equip $X$ with the following topology: (a) every $\alpha \in \omega_{2}$ is isolated; (b) an open neighborhood base for $\omega_{2}$ is $\left\{X \backslash \alpha: \alpha \in \omega_{2}\right\}$. Then every subspace of size $\omega_{1}$ of $X$ is discrete, but $X$ does not have a point-countable base. This example shows that in Theorem 1.5, $d(X)=\omega_{1}$ cannot be dropped.

Example 4.2. It is consistent to assume ([KV], p. 864) that there is an $S \subset\{\alpha \in$ $\left.\omega_{2}: \operatorname{cf}(\alpha)=\omega\right\}$ which stationary in $\omega_{2}$, but $S \cap \alpha$ is non-stationary for every limit ordinal $\alpha<\omega_{2}$. Then every subspace of cardinality $\leq \omega_{1}$ of $S$ has a point-countable base, but by the Pressing Down Lemma, $S$ does not. This shows that even in first countable spaces, $d(X)=\omega_{1}$ cannot be dropped from Theorem 1.5.

One can ask whether $d(X)=\omega_{1}$ can be removed from Theorem 3.4. The answer is consistently "yes" for locally compact spaces.

Theorem 4.3 ([B]). Under Axiom $R$, if every subspace $Y \in[X] \leq \omega_{1}$ of a locally compact space $X$ has a point-countable base, then $X$ is metrizable.

On the other hand, the answer is "no" in some other models of set theory.

Example 4.4. S. Shelah $[\mathrm{S}]$ gave a consistent example of a locally compact normal Moore space $X$ which is $\leq \omega_{1}$-collectionwise Hausdorff but not $\omega_{2}$-collectionwise Hausdorff. These properties imply that every subspace of size $\leq \omega_{1}$ of $X$ has a point-countable base, but $X$ does not.

Example 4.5. Let $\left\langle H_{i}\right\rangle_{i \in I}$ be a maximal uncountable family of uncountable subsets of $\omega_{1}$ such that $i \neq j$ in $I$ implies that $H_{i} \cap H_{j}$ is countable. Pick $I$ so that $I \cap \omega_{1}=\emptyset$ and note that by maximality, $|I| \geq \omega_{2}$. Let us consider the space $X$ with underlying set $I \cup \omega_{1}$, each point in $\omega_{1}$ isolated and each $i \in I$ having $Y_{i}=\left\{H_{i} \backslash E: E \in\left[\omega_{1}\right]^{\omega}\right\}$ as an open neighborhood basis. Then $X$ is a zero-dimensional $T_{2}$-space, each subspace $Y \in[X]^{\omega_{1}}$ is meta-Lindelöf (even ultraparacompact), but $X$ is not meta-Lindelöf, because $d(X)=\omega_{1}$ and $L(X) \geq \omega_{2}$.

Thus in Theorem 2.4 we cannot $\operatorname{relax} " X=\bigcup\left\{\bar{A}: A \in[D]^{\omega}\right\}$ for some $D \in$ $[X] \leq \omega_{1} "$ to $d(X)=\omega_{1}$.

Example 4.6. Assume that $2^{\omega} \geq \omega_{2}$. Let $A$ be a countable dense subset of the Tychonoff cube $I^{\omega_{2}}$. By the Tychonoff Embedding Theorem, $I^{\omega_{2}}$ contains a copy of the ordinal space $\omega_{2}$ that we will also denote by $\omega_{2}$. Consider the subspace $X=A \cup \omega_{2}$ of $I^{\omega_{2}}$. Then every $Y \in[X]^{\omega_{1}}$ is meta-Lindelöf (even Lindelöf) in $X$, $d(X)=\omega$ and yet $X$ is not meta-Lindelöf.

This example shows that $L(X)=\omega_{1}$ cannot be dropped from Corollary 2.7. 
Question 4.7. Is it consistent that if $X$ is a first countable space and every $Y \in$ $[X] \leq \omega_{1}$ has a point-countable base, then $X$ has a point-countable base?

By Example 4.2, consistency is the best we can hope for.

Question 4.8. Suppose $X$ is a paracompact $p$-space and every $Y \in[X]]^{\leq \omega_{1}}$ has a point-countable base. Is $X$ metrizable?

By Theorem 3.4, the answer is yes, if $d(X)=\omega_{1}$.

Question 4.9. Suppose $X$ is a Lindelöf space, and every $Y \in[X]^{\omega_{1}}$ has a pointcountable base. Does $X$ have a point-countable base?

Question 4.10. Is it consistent that if $X$ is a first countable space and every $Y \in[X]^{\leq \omega_{1}}$ is meta-Lindelöf, then $X$ is meta-Lindelöf?

Again by Example 4.2, consistency is the best to expect.

Question 4.11. Suppose $d(X) \leq \omega_{1}$ and every $Y \in[X] \leq \omega_{1}$ is submeta-Lindelöf. Is $X$ submeta-Lindelöf?

\section{REFERENCES}

[B83] Z. Balogh, Locally nice spaces under Martin's Axiom, Comment. Math. Univ. Carolin. 24 (1983), 63-87. MR 85b:54005

[B] Z. Balogh, Locally nice spaces and Axiom $R$ (to appear).

[D88] A. Dow, An empty class of nonmetric spaces, Proc. Amer. Math. Soc. 104 (1988), 999-1001. MR 89j:54032

[D91] A. Dow, An introduction to applications of elementary submodels to topology, Topology Proc. 13 (1988), 17-72. MR 91a:54003

[HV] R.E. Hodel, J.E. Vaughan, Reflection theorems for cardinal functions, Topology Appl. 100 (2000), 47-66. MR 2001b:54004

[K] K. Kunen, Set theory, North-Holland, 1980. MR 82f:03001 Reprint MR 85e:03003

$[\mathrm{KV}]$ K. Kunen and J.E. Vaughan, eds., Handbook of Set-theoretic Topology, North-Holland, 1984. MR 85k:54001

[S] S. Shelah, Remarks on $\lambda$-collectionwise Hausdorff spaces, Topology Proc. 2 (1977), 583-592. MR 81f:03060

[V] J.E. Vaughan, On Dow's reflection theorem for metrizable spaces, Topology Proc. 22 (1997), 351-361. MR 99m:54018

Department of Mathematics \& Statistics, Miami University, Oxford, Ohio 45056

E-mail address: baloghzt@muohio.edu 